\title{
Medulloblastoma: From Origin to Treatment
}

\section{ISSN: 2637-773X}

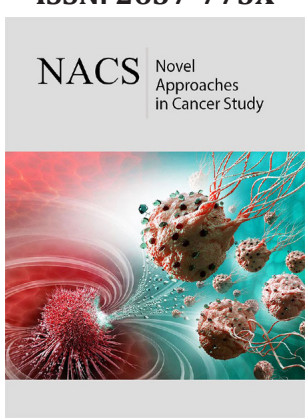

*Corresponding author: Yi Lu, Department of Pathology and Laboratory Medicine, University of Tennessee Health Science Center, Cancer Research Building, Room 258, 19 South Manassas Street, Memphis, TN 38163, USA, Email: ylu@uthsc.edu

Submission: 侮 March 12, 2020

Published: 泚June 10, 2020

Volume 4 - Issue 5

How to cite this article: Daniel Chizhikov, Chikezie O Madu, Yi Lu. Medulloblastoma: From Origin to Treatment. Nov Appro in Can Study. 4(5). NACS.000599. 2020. DOI: 10.31031/NACS.2020.04.000599

Copyright@ Yi Lu, This article is distributed under the terms of the Creative Commons Attribution 4.0 International License, which permits unrestricted use and redistribution provided that the original author and source are credited.

\author{
Daniel Chizhikov ${ }^{1,2}$, Chikezie $0 \mathrm{Madu}^{2}$ and Yi Lu${ }^{3 *}$
}

${ }^{1}$ School of Health Studies, University of Memphis, Memphis, TN 38152, USA, danielchizhikov@ gmail.com

${ }^{2}$ Departments of Biology and Advanced Placement Biology, White Station High School, Memphis, Tennessee, 38117, USA, maduco@scsk12.org

${ }^{3}$ Department of Pathology and Laboratory Medicine, University of Tennessee Health Science Center, Memphis, TN 38163, USA, ylu@uthsc.edu

\begin{abstract}
Medulloblastoma is the most frequent childhood brain tumor. Medulloblastoma affects the cerebellum, the brain region that regulates motor coordination and is also involved in certain cognitive functions. Medulloblastoma is a heterogeneous tumor, and recent advances in high-throughput genomic techniques have allowed medulloblastoma to be classified into four molecularly distinct subgroups: the SHHgroup, WNT-group, group 3, and group 4 medulloblastoma. Different medulloblastoma subgroups are characterized by distinct molecular alterations, are believed to originate from distinct types of neural progenitors, and have different clinical outcomes. WNT and SHH groups are relatively well understood and are named so because the WNT and SHH signaling pathways play a key role in their development. In contrast, molecular alterations and developmental mechanisms of group 3 and 4 medulloblastoma remain largely unknown. Although the survival of medulloblastoma patients has improved over the last 30 years, reaching the 5-year survival rate of $60-80 \%$, treatment of this type of tumor is still associated with severe side effects. A deeper understanding of medulloblastoma biology and developing new pathway-specific therapies is urgently required for better treatment of medulloblastoma patients.
\end{abstract}

Keywords: Medulloblastoma; Cerebellum; Development; Medulloblastoma subgroups; Medulloblastoma pathways

\section{Introduction}

Medulloblastoma is a brain tumor, which is most frequently diagnosed in children [1,2]. The term "medulloblastoma" was introduced by Drs. Cushing and Bailey in 1925 to describe childhood tumors located in the posterior fossa, a cerebrospinal fluid- filled space located at the back of the skull [3]. During the following decades, it was determined that most medulloblastoma cases originate in the cerebellum, the brain region that is responsible for motor-coordination and certain higher order cognitive functions. Traditionally, medulloblastoma was classified based on histology. In 2016, a new classification of medulloblastoma, which better reflects its pathology and prognosis, was introduced. This classification is largely based on molecular alterations observed in medulloblastoma tumors and subdivides all medulloblastoma cases into four groups: the SHH group, WNT group, and groups 3 and 4 [4].

Growing evidence indicates that medulloblastoma arises when genetic and epigenetic alterations compromise the normal cerebellar developmental program, in particular the proliferation and differentiation of cerebellar progenitors and stem cells [5]. It is believed that each group of medulloblastoma arises from a distinct type of cerebellar progenitors and is driven by specific genetic and epigenetic alterations [6]. While well established for some medulloblastoma groups (such as for the SHH group and to a lesser degree for the WNT group), the developmental origin and molecular mechanisms for other medulloblastoma types are poorly understood [7-9]. 
Despite the advancement of medulloblastoma treatment protocols, the 5-year survival rate of medulloblastoma patients does not typically exceed $70 \%$, and medulloblastoma treatment remains to be associated with significant side effects [3]. A better understanding of the mechanisms that drive medulloblastoma formation is clearly important for the development of more efficient medulloblastoma treatment strategies. In this review, we will discuss the cellular and molecular mechanisms that regulate normal cerebellar development and how their disruption leads to the development of specific types of medulloblastoma.

\section{Cerebellar development}

During development, the cerebellar anlage arises from the alar plate (dorsal region) of rhombomere 1 in the anterior hindbrain [10]. Because of the limited availability of human embryonic tissue, most of our knowledge about cerebellar neurogenesis came from the analysis of model organisms, particularly mice. It is believed, however, that cerebellar development is largely conserved between humans and mice, and, thus, key developmental mechanisms identified in mice are also applicable to the human cerebellum $[11,12]$.

Once established during early embryogenesis, the cerebellar anlage harbors two germinal zones (progenitor domains): the cerebellar ventricular zone and cerebellar rhombic lip (Figure 1).
The cerebellar ventricular zone produces GABAergic cerebellar neurons, including Purkinje neurons [13]. Purkinje neurons exit the ventricular zone largely as post-mitotic cells and migrate radially from the site of their origin. Another cellular population that arises from the cerebellar ventricular zone are astroglial like progenitors. After exiting the cerebellar ventricular zone, astroglial-like progenitors populate cerebellar parenchyma and later cerebellar white matter, where they proliferate and eventually produce GABAergic interneurons, cerebellar glia, and, likely, also give rise to stem cells that continue populating the cerebellar white matter. The cerebellar rhombic lip is the initial place of origin of all glutamatergic (excitatory) neurons of the cerebellum, such as granule cells and neurons of cerebellar nuclei [14]. Glutamatergic cerebellar neurons exit the rhombic lip during early embryonic development and tangentially migrate to the nuclear transitory zone before forming deep cerebellar nuclei. Then, the rhombic lip begins producing granule cell precursors that generate the external granule layer (EGL), which is a secondary germinal zone in the late embryonic and early postnatal cerebellum (Figure 1) [14,15]. Granule cells are the most abundant neurons in the entire brain and account for $80 \%$ of all neurons in humans and $60 \%$ of neurons in mice. Thus, dramatic expansion of granule precursors in the EGL due to proliferation is necessary to produce enough mature granule neurons to establish proper cerebellar circuits [12].

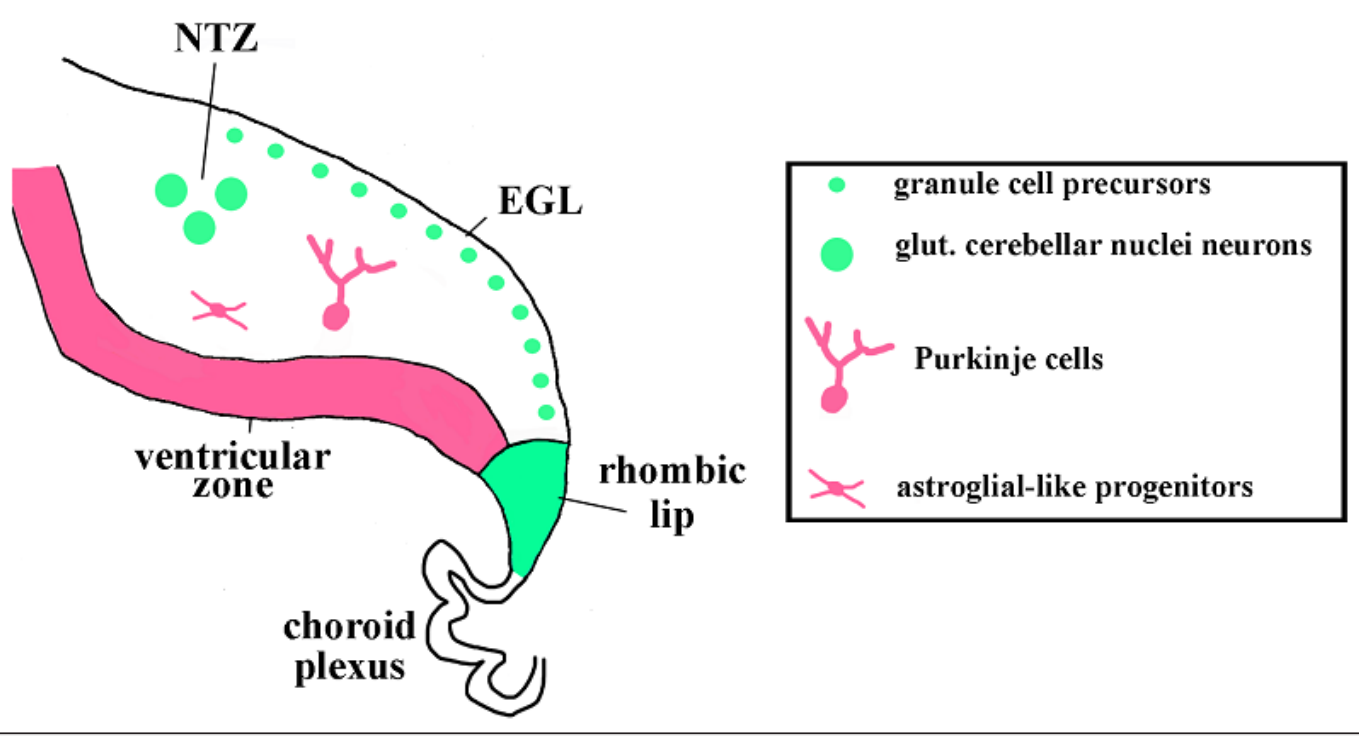

Figure 1: Key cell types and their origin in the developing cerebellum.

The sagittal section of the developing cerebellum is shown. The external granule cell layer (EGL) and nuclear transitory zone (NTZ) are labeled. Glutamatergic cerebellar neurons (cerebellar nuclei neurons and granule precursors) originate from the rhombic lip (green), which is located adjacent to the choroid plexus. GABAergic cerebellar cell types (Purkinje cells and astroglial-like progenitors that produce molecular layer interneurons) originate from the ventricular zone (pink).

During late embryogenesis (around postnatal days 1-3 in mice), Purkinje cells begin forming a monolayer below the EGL. They secrete sonic hedgehog $(\mathrm{SHH})$ protein, a potent mitogen for granule cell precursors, thus, regulating the proliferation and expansion of granule cells (Figure 2) [16-19]. After exiting the cell cycle and beginning differentiation, granule cells migrate inward along Bergmann glial fibers. This leads to the disappearance of the EGL and the formation of the internal granule cell layer (IGL) beneath 
the Purkinje cell layer. In mice, the EGL disappears by postnatal day 16 , but in humans the EGL persists much longer, and is still detected one year after birth [20].

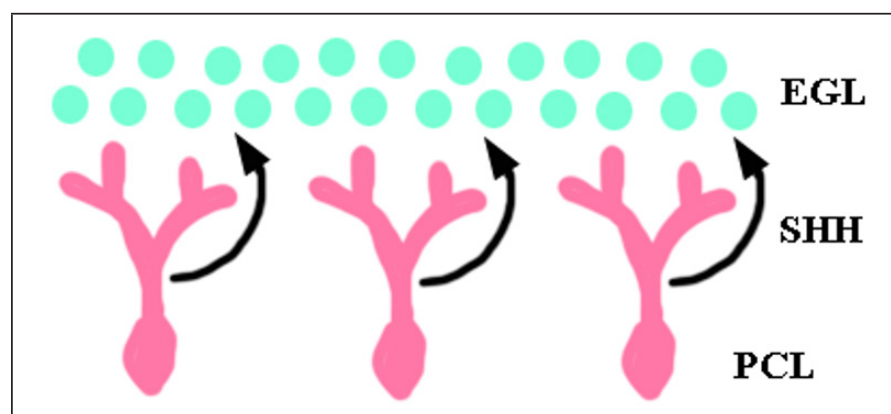

Figure 2: Purkinje cells secrete SHH to promote the proliferation of progenitors in the EGL.

Purkinje cells are pink, granule cell precursors are green. PCL - Purkinje cell layer. Arrows indicate SHH protein secreted by Purkinje cells that reaches granule cell precursors.

More than a hundred genes are known to regulate cerebellar development. Since the genetic regulation of cerebellar development has been the focus of several recent reviews $[11,21,22]$, this topic will only be briefly touched in this paper. Probably the most wellknown transcription factors that regulate cerebellar development are Atoh1, Pax6 and Zic1. They are expressed in granule cell precursors in the EGL and regulate the migration, proliferation and survival of these cells [23-25]. N-Myc, C-Myc, and Gli also regulate the proliferation of cerebellar progenitors, particular granule precursors in the EGL [26,27]. Wnt signaling, a well-known mitogen for many types of cells, promotes the progenitor expansion in the ventricular zone, cerebellar parenchyma and white matter. Interestingly, Wnts do not promote expansion of granule cell precursors, highlighting the difference in the molecular networks that operate in distinct progenitor domains [28].

\section{Medulloblastoma: Epidemiology, developmental origin, and molecular pathways}

Most medulloblastoma cases are diagnosed in children younger than nine years old, although medulloblastoma can also develop in adults, typically those younger than 40 years. Most medulloblastoma cases are sporadic, and fewer than 5\% are associated with familial cancer predisposition syndromes. Boys have a slightly higher frequency of medulloblastoma than girls, and no significant difference in medulloblastoma incidence was reported between different races and ethnic groups [1-3].

In contrast to many other human cancers, no clear association with non-genetic risk factors, such as environmental exposures, has been identified for medulloblastoma. Similar to other tumors, however, medulloblastoma arises as the result of the accumulation of oncogenic mutations and epigenetic changes in neural progenitors or stem cells [5]. The genetic and epigenetic changes found in different cases of medulloblastoma, as well as their cells of origin, significantly affect tumor behavior and prognosis, and allow the classification of medulloblastoma into four major subgroups. These four groups are discussed below, including their cells of origin and the most common mutations.

\section{SHH group}

Approximately $25 \%$ of medulloblastoma cases belong to the SHH subgroup and are characterized by an intermediate prognosis (60-80\% survival rate) [6]. Medulloblastomas of this subgroup arise from granule cell precursors in the EGL because of an overactivation of the SHH signaling pathway, an important regulator of normal granule cell development [7,8]. Different genetic events affecting the SHH pathway have been described in SHH medulloblastomas. These include mutations inactivating negative regulators of the SHH Pathway, including PTCH1 and SUFU, and mutations activating positive regulators or downstream effectors of the SHH pathway such as SMO, GLI1, GLI2, YAP1 and MYCN [29,30]. Mutations of tumor suppressor p53 have been reported as well [31].

\section{WNT group}

Approximately $10 \%$ of medulloblastomas belong to this group. It is characterized by an excellent (more than 95\%) survival rate [6]. Surprisingly, at least some tumors of this group arise from progenitors outside the cerebellum, in the lower rhombic lip - the most dorsal region of the brainstem $[32,33]$. The vast majority (up to $90 \%$ ) of WNT medulloblastomas possess mutations activating the CTNB1 gene that encodes beta-catenin, the downstream mediator of the WNT signaling pathway [30]. Activated beta-catenin enters the nucleus and activates the expression of its downstream genes, many of which are positive regulators of the cell cycle, and thus, promote proliferation of medulloblastoma cells. Loss-of-function mutations of APC, an inhibitor of the WNT signaling pathway, were discovered in the WNT group of medulloblastoma as well [34].

\section{Group 3}

This is the most aggressive type of medulloblastoma (survival rate is less than $60 \%$ ). Approximately $25 \%$ of medulloblastomas belong to this group [6]. Group 3 medulloblastomas are characterized by the amplification and overexpression of MYCN but not other SHH-related genes [31]. Alterations in the NOTCH signaling pathway, transcription factor OTX2, and multiple proteins involved in transcription and translation have also been reported in this type of medulloblastoma [35]. The cellular origin of group 3 medulloblastoma is poorly understood, but it is believed that this type of medulloblastoma arises from ventricular zone progenitors or other progenitors in the embryonic or neonatal cerebellum [59].

\section{Group 4}

This is the most common but least understood group of medulloblastoma. It is characterized by an intermediate prognosis. Mutations and the misregulation of expression of multiple genes have been described in group 4 medulloblastoma, but none of the genes are mutated in more than $10 \%$ of cases [6,30]. Recent gene expression studies suggest that Group 4 medulloblastomas originate from glutamatergic deep cerebellar nuclei progenitors, possibly those located in the early cerebellar rhombic lip [9]. 


\section{Medulloblastoma treatment}

Medulloblastoma treatment involves a combination of radiation therapy, surgery, and chemotherapy [36]. Conventional medulloblastoma treatment results in $\sim 70 \%$ of the overall fiveyear survival rate but frequently leads to severe life-long side effects, which include the disruption of the endocrine system, neurocognitive impairment, and the formation of secondary tumors [37-40]. Work on improving medulloblastoma treatment involves several areas, including optimization of surgery, radiotherapy optimization, and the development of targeted therapies that focus on the molecular pathways that drive medulloblastoma development. Several drugs targeting specific molecular pathways are currently in clinical trial. For example, a SMO inhibitor known as Vismodegib may improve the treatment of $\mathrm{SHH}$ subgroup medulloblastoma [36,41]. Unfortunately, little progress has been made in the development of targeted therapy for the most aggressive or most common medulloblastoma groups (groups 3 and 4). No direct suitable inhibitor for MYCN to target group 3 medulloblastoma and no predominant molecular target for group 4 medulloblastoma have been identified yet.

Another attractive option for medulloblastoma treatment is targeting tumor-initiating stem cells. Although radiation and chemotherapy are effective against rapidly proliferating tumor cells, they are not very effective against slowly proliferating medulloblastoma-initiating stem cells, which may be largely responsible for tumor relapse and dissemination [5,42]. The development of agents against medulloblastoma-initiating stem cells is prevented by a poor understanding of the biological nature of these cells.

\section{Conclusion}

During the last two decades, significant progress has been made in our understanding of the molecular and cellular mechanisms that drive medulloblastoma formation, in particular the SHH and WNT groups of medulloblastomas. In contrast, the most aggressive and/ or most common medulloblastoma, groups 3 and 4, remain poorly understood. A better understanding of medulloblastoma biology is expected to help develop more efficient treatment strategies that will improve patients' survival rates and reduce side effects.

\section{Competing Interests}

The authors have declared that no competing interest exists.

\section{Acknowledgement}

We are grateful to Pranav Rajashekar and Sarah Ding for critical comments related to the manuscript.

\section{References}

1. Ferlay J, Soerjomataram I, Ervik M, Dikshit R, Eser S, et al. (2015) Cancer incidence and mortality worldwide: sources, methods and major patterns in GLOBOCAN 2012. Int J Cancer 136(5): E359-E386.

2. Patel S, Bhatnagar A, Wear C, Osiro S, Gabriel A, et al. (2014) Are pediatric brain tumors on the rise in the USA? Significant incidence and survival findings from the SEER database analysis. Childs Nerv Syst 30(1): 147154.
3. Nathan, Kevin C (2016) Medulloblastoma. J Child Neurol 31(12): 13411353.

4. Louis DN, Perry A, Reifenberger G, von Deimling A, Branger D, et al. (2016) The 2016 World Health Organization classification of tumors of the central nervous system: A summary. Acta Neuropathol 131(6): 803820 .

5. Azzarelli R, Simons BD, Philpott A (2018) The developmental origin of brain tumours: A cellular and molecular framework. Development 145(10): 1-12.

6. Northcott PA, Robinson GW, Kratz CP, Mabbott DJ, Pomeroy SL, et al. (2019) Medulloblastoma. Nat Rev Dis Primers 5(1): 11.

7. Schüller U, Heine VM, Mao J, Kho AT, Dillon AK, et al. (2008) Acquisition of granule neuron precursor identity is a critical determinant of progenitor cell competence to form Shh-induced medulloblastoma. Cancer Cell 14(2): 123-134.

8. Yang ZJ, Ellis T, Markant SL, Read TA, Kessler JD, et al. (2008) Medulloblastoma can be initiated by deletion of patched in lineagerestricted progenitors or stem cells. Cancer Cell 14(2): 135-145.

9. Lin CY, Erkek S, Tong Y, Yin L, Federation AJ, et al. (2016) Active medulloblastoma enhancers reveal subgroup-specific cellular origins. Nature 530(7588): 57-62.

10. Haldipur P, Dang D, Millen KJ (2018) Embryology. Handb Clin Neurol 154: 29-44.

11. Leto K, Arancillo M, Becker EB, Buffo A, Chiang C, et al. (2016) Consensus paper: Cerebellar development. Cerebellum 15(6): 789-828.

12. Haldipur P, Millen KJ (2019) What cerebellar malformations tell us about cerebellar development. Neurosci Lett 688: 14-25.

13. Hoshino M, Nakamura S, Mori K, Kawauchi T, Terao M, et al. (2005) Ptf1a, a bHLH transcriptional gene, defines GABAergic neuronal fates in cerebellum. Neuron 47(2): 201-213.

14. Machold R, Fishell G (2005) Math1 is expressed in temporally discrete pools of cerebellar rhombic-lip neural progenitors. Neuron 48(1): 1724.

15. Wang VY, Rose MF, Zoghbi HY (2005) Math1 expression redefines the rhombic lip derivatives and reveals novel lineages within the brainstem and cerebellum. Neuron 48(1): 31-43.

16. Wechsler-Reya RJ, Scott MP (1999) Control of neuronal precursor proliferation in the cerebellum by Sonic Hedgehog. Neuron 22(1): 103114.

17. Lewis PM, Gritli-Linde A, Smeyne R, Kottmann A, McMahon AP (2004) Sonic hedgehog signaling is required for expansion of granule neuron precursors and patterning of the mouse cerebellum. Dev Biol 270(2): 393-410.

18. Corrales JD, Rocco GL, Blaess S, Guo Q Joyner AL (2004) Spatial pattern of sonic hedgehog signaling through Gli genes during cerebellum development. Development 131(22): 5581-5590.

19. Heil C (2019) Hedgehog pathway permissive conditions allow generation of immortal cell lines from granule cells derived from cancerous and non-cancerous cerebellum. Open Biol 9(1): 180145.

20. Wang VY, Zoghbi HY (2001) Genetic regulation of cerebellar development. Nat Rev Neurosci 2(7): 484-491.

21. Joyner AL (2016) From cloning neural development genes to functional studies in mice, 30 years of advancements. Curr Top Dev Biol 116: 501515 .

22. Wang L, Liu Y (2019) Signaling pathways in cerebellar granule cells development. Am J Stem Cells 8(1):1-6.

23. Ben-Arie N, Bellen HJ, Armstrong DL, McCall AE, Gordadze PR, et al. (1997)Math1 is essential for genesis of cerebellar granule neurons. Nature 390(6656): 169-172. 
24. Flora A, Klisch TJ, Schuster G, Zoghbi HY (2009) Deletion of atoh1 disrupts sonic hedgehog signaling in the developing cerebellum and prevents medulloblastoma. Science 326(5958): 1424-1427.

25. Castellino RC, Kenney AM (2019) Atoh1/MATH1 adds up to ciliogenesis for transducing SHH signaling in the cerebellum. Dev Cell 48(2): 129130.

26. Zindy F, Knoepfler PS, Xie S, Sherr CJ, Eisenman RN, et al. (2006) N-Myc and the cyclin-dependent kinase inhibitors p18Ink4c and p27Kip1 coordinately regulate cerebellar development. Proc Natl Acad Sci USA 103(31): 11579-11583.

27. Wey A, Martinez Cerdeno V, Pleasure D, Knoepfler PS (2010) C- and $\mathrm{N}$-myc regulate neural precursor cell fate, cell cycle, and metabolism to direct cerebellar development. Cerebellum 9(4): 537-547.

28. Pei Y, Brun SN, Markant SL, Lento W, Gibson P, et al. (2012) WNT signaling increases proliferation and impairs differentiation of stem cells in the developing cerebellum. Development 139(10): 1724-1733.

29. Kool M, Jones DT, Jäger N, Northcott PA, Pugh TJ, et al. (2014) Genome sequencing of $\mathrm{SHH}$ medulloblastoma predicts genotype-related response to smoothened inhibition. Cancer Cell 25(3): 393-405.

30. Northcott PA, Buchhalter I, Morrissy AS, Hovestadt V, Weischenfeldt J, et al. (2017) The whole-genome landscape of medulloblastoma subtypes. Nature 547(7663): 311-317.

31. Northcott PA, Shih DJ, Peacock J, Garzia L, Morrissy AS, et al. (2012) Subgroup-specific structural variation across 1,000 medulloblastoma genomes. Nature 488(7409): 49-56.

32. Gibson P, Tong Y, Robinson G, Thompson MC, Currle DS, et al. (2010) Subtypes of medulloblastoma have distinct developmental origins. Nature 468(7327): 1095-1099.

33. Phoenix TN, Currle DS, Robinson G, Gilbertson RJ (2012) Review: developmental origins of neural tumours: old idea, new approaches. Neuropathol Appl Neurobiol 38(3): 222-227.
34. Waszak SM, Northcott PA, Buchhalter I, Robinson GW, Sutter C, et al. (2018) Spectrum and prevalence of genetic predisposition in medulloblastoma: A retrospective genetic study and prospective validation in a clinical trial cohort. Lancet Oncol 19(6): 785-798.

35. Archer TC, Ehrenberger T, Mundt F, Gold MP, Krug K, et al. (2018) Proteomics, post-translational modifications, and integrative analyses reveal molecular heterogeneity within medulloblastoma subgroups. Cancer Cell 34(3): 396-4101.

36. Thomas A, Noël G (2019) Medulloblastoma: optimizing care with a multidisciplinary approach. J Multidiscip Healthc 12: 335-347.

37. Law N, Smith ML, Greenberg M, Bouffet E, Taylor MD, et al. (2017) Executive function in paediatric medulloblastoma: The role of cerebrocerebellar connections. J Neuropsychol 11(2): 174-200.

38. Moxon-Emre I, Bouffet E, Taylor MD, Laperriere N, Scantlebury N, et al. (2014) Impact of craniospinal dose, boost volume, and neurologic complications on intellectual outcome in patients with medulloblastoma. J Clin Oncol 32(17): 1760-1768.

39. Moxon-Emre I, Taylor MD, Bouffet E, Hardy K, Campen CJ, et al. (2016) Intellectual outcome in molecular subgroups of medulloblastoma. J Clin Oncol 34(34): 4161-4170.

40. Gerber NU, Mynarek M, von Hoff K, Friedrich C, Resch A, et al. (2014) Recent developments and current concepts in medulloblastoma. Cancer Treat Rev 40(3): 356-365.

41. Robinson GW, Orr BA, Wu G, Gururangan S, Lin T, et al. (2015) Vismodegib exerts targeted efficacy against recurrent sonic hedgehogsubgroup medulloblastoma: Results from phase II pediatric brain tumor consortium studies PBTC-025B and PBTC-032. J Clin Oncol 33(24): 2646-2654.

42. Huang GH, Xu QF, Cui YH, Li N, Bian XW, et al. (2016) Medulloblastoma stem cells: Promising targets in medulloblastoma therapy. Cancer Sci 107(5): 583-589. 